\title{
NOTE ON THE SHORT-CIRCUITING OF CADMIUM CELLS.
}

By P. I. Wold.

SEVERAL of the cadmium cells mentioned in a previous article ${ }^{1}$ $S$ have been short-circuited for different lengths of time to find the rate at which they recover and some of the recovery curves obtained are given below.

The period of short-circuiting varied from 15 seconds to 10 minutes and the general shape of the curves is the same in all cases and agrees very well with the curves obtained in a similar manner by F. E. Smith ${ }^{2}$ and by Barnett. ${ }^{3}$

The internal resistance of these cells is about $1,500 \mathrm{ohms}$ so that the actual current while short-circuited is rather small. The deviation from the normal value was obtained by a Wolff potentiometer, the short-circuited cell being placed in opposition to a cell taken as a standard and their differences read. Deviations from the normal were read directly to $1 / 100,000$ of a volt and were estimated, with a fair degree of accuracy, to $1 / 10$ of this.

The curves shown are typical curves out of a large number.

Fig. I shows the close agreement between two cells, 44 and 45 , both short-circuited for 15 seconds. The cell 45 was shortcircuited five times for a period of I 5 seconds and the recovery curves were identically the same to within the width of a line.

Fig. 2 shows the effect of short-circuiting cell 44 differen tlengths of time. Curve $I$ is for 15 seconds, curve 2 for 30 seconds, curve 3 for 60 seconds, curve 4 for 2 minutes, curve 5 for 5 minutes and curve 6 for 10 minutes.

In these two figures the axis $O A$ represents the normal value of the E.M.F. of the cells and the ordinates represent the deviation from this normal in hundred-thousandths of a volt.

${ }^{1}$ Physical Review, vol. 27, p. 329, 1908.

${ }^{2}$ Philosophical Transactions of the Royal Society of London; Series A, vol. 207, p. 393.

${ }^{3}$ Physical Review, vol. 18, p. 104, 1904. 


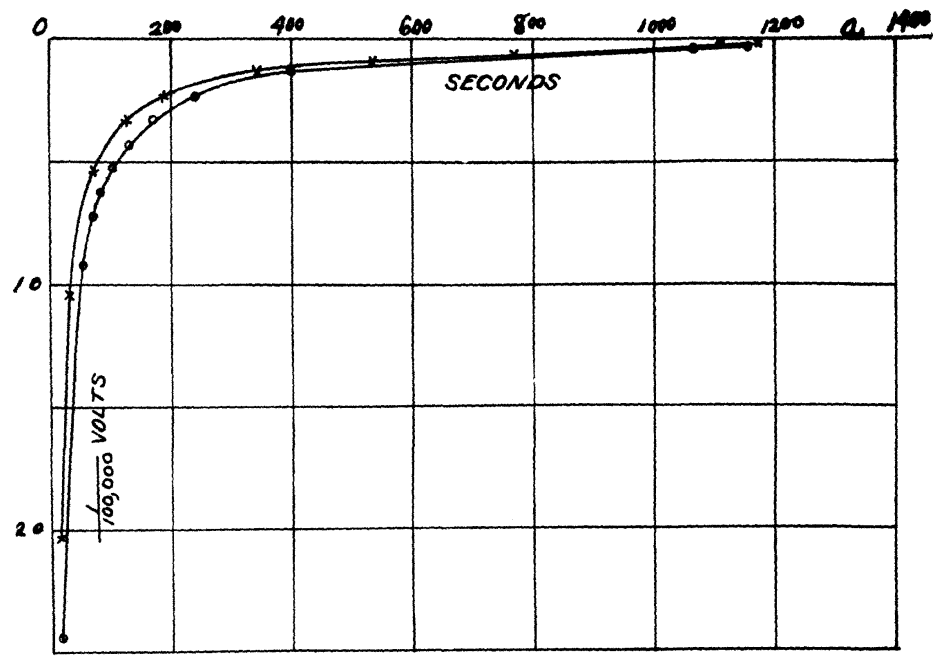

Fig. 1.

In his work Barnett subjected his cells to a much more severe test than in the present case, but apparently in this case the author was able to carry the measurements one place further in the decimal. Even with this increased accuracy in readings, the points, as plotted, fall remarkably close to the curve, indicating, to this degree of re-

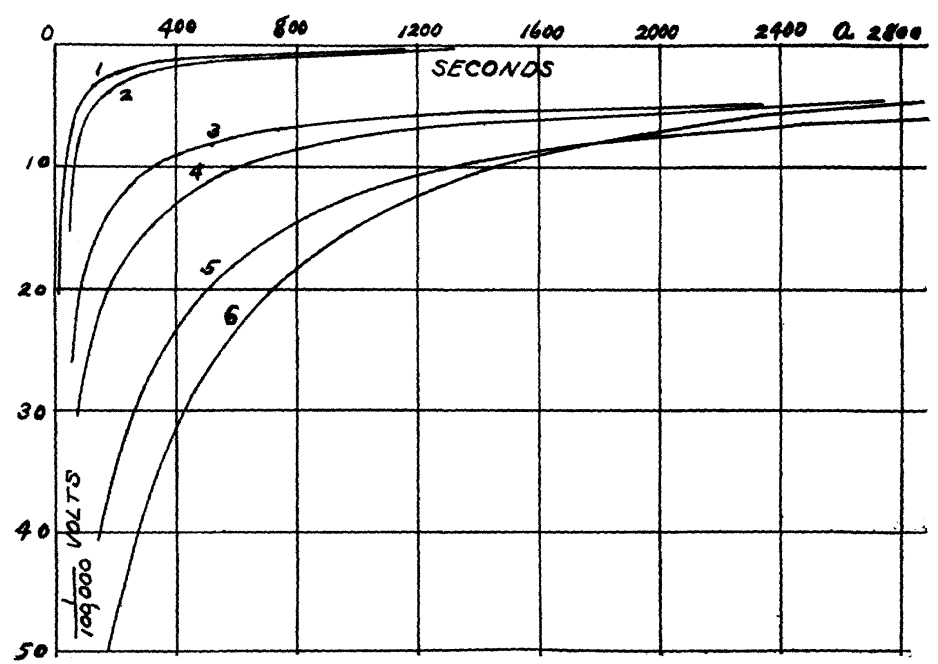

Fig. 2. 
finement, a very definite and smooth recovery curve free from accidental disturbances within the cell itself.

An attempt to find a general form of equation for the recovery curve has, so far, been unsuccessful. The form suggests a logarithmic curve, but plotting logarithms of the deviation with time fails to give even an approximately straight line.

The general similarity of the decay curves in the case of phosphorescence, as shown by Professor Nichols and Merritt, with these recovery curves then suggested that these two effects might obey similar laws since in both cases the curve is probably determined by the reunion of free particles; in the one case probably electrons, in the other case the chemical ions which cause the polarization.

In their work on phosphorescence ${ }^{1}$ Professors Nichols and Merritt have concluded that the curve obtained by plotting the reciprocal of the square root of the intensity with time is a straight line for small values of $t$ and that it changes to a curve concave to the axis of $t$ as $t$ increases; but for larger values of $t$ the relation between $I^{-\frac{1}{2}}$ and $t$ is again linear, the two straight portions merging into each other.

The results when the values of $D^{-\frac{1}{2}}$ and $t$, as given in the curves of Fig. 2, were plotted as shown in Fig. 3, the numbers of these curves corresponding to the numbers in Fig. 2. In this case $D$ represents the intensity or amount of polarization, $i . e$., the deviation of the cell from normal E.M.F. The curves agree in form surprisingly well with the corresponding curves shown by Professors Nichols and Merritt.

Because of the difficulties in obtaining readings in the very first part of the recovery it cannot be said at present that the first part of the above curves would also be straight lines with a different slope. Probably the shape of this curve is due to the fact that there are two, or more, effects giving or intensifying the polarization from which the cell must recover, and these individual recoveries are of different velocities. It may be that one phase of the recovery is brought about by the diffusion of ions, which at first are concentrated close to a separating surface in the cell, and the other part of the recovery is due to the reunion of these ions. At first probably

${ }^{1}$ Physical Review, vol. 22, p. 279, 1906 ; vol. 23, p. 37, 1905. 
the diffusion effect would be the predominant one, following one law, but later the chief effect would be that due to reunion of ions, which would follow a different law, similar perhaps to the law governing the reunion of electrons in the case of phosphorescence.

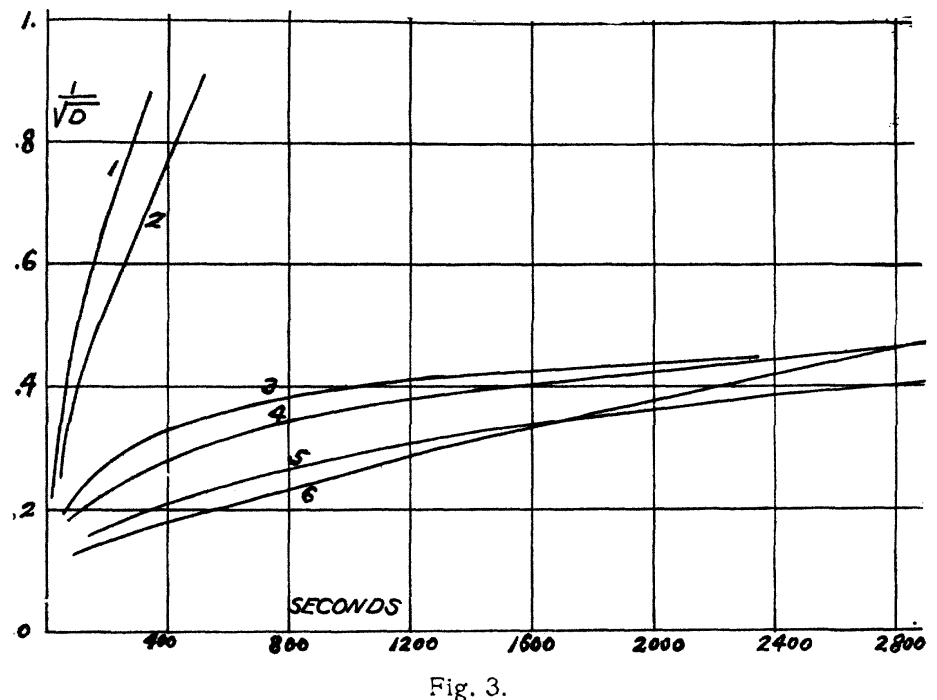

It may be, on the other hand, that we have simply the case of an excess of ions, producing polarization, at different points; as $\mathrm{SO}_{4}$ ions near the cadmium and cadmium ions near or in the $\mathrm{HgSO}_{4}$ and that the rate of recovery from these effects is different.

The author hopes to be able to do more work on this matter with the idea of determining with which case we have to deal.

Physical Laboratory,

Cornell University. 